https://doi.org/10.48009/1_iis_2005_59-65

\title{
ADAPTABILITY OF COLA FRAMEWORK
}

\section{Alexander Vengerov, Ramapo College of NJ, abvenger@ ramapo.edu}

\begin{abstract}
The ubiquitous need for learning with resulting adaptation to change has led to the dramatic variability in types of learners, learning situations, and environments. Such learning is shifting to the learning environments with distributed dynamic assisting actors and processes, often not centrally controlled and not sharing the same theoretical and methodological vision of learning situations. The collaboration, group decision-making, and learner's adaptation needs require the development of a shared architectural framework capable of incorporating in its design the variety and dynamism of new learning. This paper presents the important mechanisms of a context-oriented approach supporting the adaptability and flexibility of learning architectures.
\end{abstract}

Keywords: learning, learning architecture, COLA, context, dynamic systems, complexity

\section{INTRODUCTION}

The growing sensitivity of systems in the industry, society, and even everyday life leads to the increased volume of changes and needs for adaptation and learning. This creates a new situation where learning from being purely academic finite size knowledge transfer is becoming a ubiquitous always-on service or utility continuously provided for actors of all types in their increasingly dynamic and complex situations. Learners and teachers are also not what they used to be, transforming into continuously learning and teaching systems consisting of various actors and means of automation. People are "plugged" into learning, AI, and DSS environments at home and at work. Even simplest devices are getting IP addresses, change their own functionality and have to be continuously adjusting to the environments, which they themselves modify. The speed, intensity and importance of learning is increasing dramatically.

The new type of learning situations result into a new problem of increasing cost, complexity, and variability of the learning process combined with diminishing returns of teaching or learning administration efforts. The core of this problem is in insufficient flexibility and adaptability of used resources (especially people of different persuasions and skills and information technology) that have to match the scale and dynamism of environmental changes by something like learning-on-demand or utility learning bridging the concept with utility computing set to support the necessary computing resources for any requesting it automated business process.

The necessary continuous availability-on-demand and adaptability of resources in complex and dynamic environments is a known common problem for various areas. The common solution is envisioned by IBM, SUN, HP, Microsoft and other leaders in the development of adaptive learning organizations as being based on good common virtualization allowing for quick orientation and decision making. Such virtualization or vision space should be supported by flexibility and speed of collaborative development of generally accepted views/models of emerging situations, allowing to integrate efforts in research, development, delivery, and support of educational services (effectiveness of collaboration). 
The learning model and properly virtualized architecture can help in dealing with a variety of teaching/learning actor types (human, group, machine, hybrid) and learning situations (in-class, life-long, work-based, project-oriented, system-supported, etc.). This paper is focusing on the importance and methods supporting adaptability of learning architectures to changing learning situation and it's analysis. We will also show that the explicit context consideration along with virtualized space of COLA concepts and components can serve as an effective unified framework for adaptive collaborative learning management.

\section{PART 1. ADEQUACY OF LEARNING ARCHITECTURES}

Learning architectures are based on virtualized system of interrelated conceptual representations supporting shared vision of learning processes, entities, and results, and allowing for collaborative efforts in maintaining the effectiveness and efficiency of managed learning. Adequacy and adaptability of learning architectures allow for effective cooperation and collaboration among distributed participants of all types. In addition to the possibility of quickly establishing common vision for further analysis and decision-making, they have to be able to flexibly change views of various parts and aspects of learning to more general and abstract or more fitting specific technical or pedagogical approach supporting richness of perception and constructiveness of discussions and corrective actions.

The effectiveness and efficiency of supporting actions and processes in learning environments performed by distributed actors of all kinds of cultural perceptions, technological experiences, and methodological persuasions is usually based on the following approaches:

1. Improving collaboration by standardization of learning architectures and enforcing common vision, macro design, and terminology for all participants. This is usually done on very high levels of abstraction allowing for the differences to fit very basic and stable components like in case of a series of learning standards introduced by LTSC [1].

2. Using models and meta languages as the systems' control core, like in MDA approach used in IMS, ADL, and EML contributions to the model of learning design [2]. This allows to simplify control by modifying the models when necessary. In this case the problem shifts to such design of a set of models that all will agree on strategically and will be able to effectively agree on during tactical model modifications.

3. Using dynamic compositionality of complex workflows combined with high level description of interprocess communications, and abstracting from the details of internal process models like in service-oriented architectures.

In the context of collaborative, dynamic, and distributed learning system design and maintenance the listed above methods have certain weaknesses. The standardization, by its inherent avoidance of the local and dynamic details in learning, does not support the need of periodic dynamic rethinking of what the learner is and dealing with intricacies of learner's interactions with dynamic learning situations make the common standards of seeing learner as a black box inappropriate and insufficient. The missing in standardization richness and ease of generating of new architectural descriptions for changing situations and their perceptions can be partly compensated by the generative power of grammatical means in architectural languages and metalanguages. But here we encounter the known problem of weak semantics. Metalanguages 
(like MOF), being capable to incorporate a greater variety of structural components than specialized languages, seem to be too abstract with insufficient semantic support.

In addition to the importance of the architectural descriptive power and flexibility we need the ability to discuss and manage the learner's internal learning processes (ILP) and architectures. They are necessary to allow all the groups (and systems) with differing theoretical and methodological views to collaborate in an effective and efficient way on tactical problems of learning versus strategic design of information flows to and from the learners. The serviceoriented architectures restrict such opportunity by hiding the internal models behind external service interfaces.

Another aspect of growing dynamism of learning processes is based on the use of the means of automation with some intelligent functions enhancing the quality of learning environment and learning itself. It means that some management/control decisions in learning environments will deal with the choice "to change or let self-organize" thus trusting the existing evolving processes supported by human and artificial intelligence and making management of learning less of a control problem and more of a "gardening" process. This creates a need in implementationagonistic learning architectures.

Consequently, the requirements for the next generation of learning architectures and accompanying models could be formulated as following:

1. Be sufficiently constructive (allowing for the description and handling of rich and dynamic variety of learner's states and situations)

2. Be sufficiently flexible in allowing for the diversity of views on the way knowledge arrives and is being internally represented, ranging from abrupt symbolic constructiveness to gradual connectionist evolvability.

3. Support sufficient semantic regularity as the similarity of semantic roles of same components in different designs. This feature allows for recombination, recycling, and reuse of meaning after a change has been introduced to the system

4. Support implementation independence as being agnostic to the means and nature of components and parts actually forming the learner.

\section{PART 2. ROLE OF CONTEXT IN LEARNING}

The industrialization of learning is attempting to borrow the mass production and mass distribution methods designed for prepackaged goods from the past. Meanwhile, "in the last 30 years, virtually every social science and field of humanities has moved away from rationalistic, linear ways of thinking toward an appreciation of multiple perspectives and reasoning in context" [9]. In situated cognition it was shown that learning is fundamentally situated and dynamic in its context development $[7,8]$. The necessary richness, flexibility and compositionality of knowledge patterns are based on the proper treatment of context from which these patterns evolve [3].

The process of pattern evolvability from context in dynamic situations seems to be getting in the spotlight as one of the most important parts of learning and knowledge creation. Such 
evolvability could range from low - in case of instructionist demand to just memorize the given patterns when almost no context is presented - to high in advanced constructionist applications, active research, action assembly theory, and some others. Similar developments can be seen in automated systems where new methods of context-sensitive computing started gaining ground. They include such methods as neural networks, fuzzy logic evolutionary computation, chaotic computing, immune networks, cognitive distributed human-like information processing, and a number of others [5]. For example, evolutionary and fuzzy systems supplement each rule or solution pattern with additional sensitivity to its context in a form of fitness and membership functions. The common ground for these methods is in the explicit treatment of dependency of learned knowledge patterns on their contexts. Such approach, in general, received the unifying name of soft computing [10].

Automated distributed evolving learning for human subjects is rapidly becoming a norm requiring conceptual alignment between human and machine context treatment. Hard learning, as we can call it using the similarity to hard computing, has met the same challenges as in computing: the growing importance of context and the growing learner's need in knowledge flexibility and adequacy to context changes. However, in the situation with stable and wellstructured knowledge, clear logic of causality and crisp symbolic operations on learning objects could be very effective, allowing to transfer much bigger volumes of "knowledge" than in any collaborative or/and engaged approach. This leads to the necessity of having the flexibility of gradual dynamic switching between the context treatment styles. The higher the fluidity, fuzziness, and distribution of meaning creation - the less appropriate the instructional methods coupled with prepackaged knowledge are.

The generalized concept of soft learning can consolidate the efforts in the described above search for the new generalization of learning architectures. Thus soft learning can be seen as the approach explicitly considering the role of context in coevolving dynamic pattern-context systems (DPCS) [4]. Such concept can generalize different learning models to the common virtual plane. Soft learning can provide a better fit in the new industrial, economic, and social reality reconciling efforts and solutions in machine and human learning. The common man/machine core of this concept includes an architectural framework based on the use of DPCS flexibility in the description of systems components and processes.

\section{PART 3. DESCRIPTIVE RICHNESS OF COLA}

The Context-Oriented Learning Architecture (COLA) broadly uses DPCS as its building blocks [4], which allows to satisfy the requirements of necessary descriptive richness, graduality, and flexibility of conceptual change. The full description of all COLA features [6] is beyond the scope of this paper in which we just wanted to demonstrate the methods and principles supporting high adaptability in context-oriented architectures. COLA offers a finite number of architectural aspects that could be used recursively in drilling down and rolling up levels of coarseness and generality of vision, expanding or contracting the area included into the learning situation of interest. Such features support an architectural language of high expressiveness and compositionality. Depending on the role and parameters of DPCS forming COLA, they create such specific architectural components as blocks, areas, processes, coevolutionary loops, and interpretations with corresponding roles, attributes, and interactions. Additionally, COLA 
includes the possibility of designing attributes, placing focuses, changing roles, and sequencing all these artifacts in a way allowing to map COLA states to and from the concepts and architectures related to various existing theories and methodologies [4].

The explicit context treatment as an active architectural feature of COLA determines the two major structural groups of operations: DPCS formation (resulting into the commonly accepted view of relationships among analyzed DPCS) and DPCS transformations (resulting into various decompositions of discovered DPCS).

The DPCS formation includes the following processes: determination of context scope and included architectural component types, context clusterization, and cluster reconciliation. Architectural component types in COLA support fuzzy membership in entities and channels connecting these entities. It is becoming more and more difficult to handle the challenging situation in entity-relationship types of models when relationships become entities and visa versa. This can happen gradually and has to be reflected in the structural flexibility of the architectural framework (requirement 2). The main difference between entities and channels (the concept we will use instead of relationships) is in the level of stability and ability to maintain state by entities as compared to channels. The degree and complexity of in-channel processing can grow to an extent that some channels can start gradually resembling entities with their own states and attributes.

After the approximate typization of all DPCS of interest (context scope) is agreed upon - it is possible to cluster them into patterns (consisting of more stable entities) and contexts (demonstrating higher sensitivity and lower stability). Then it is necessary to perform cluster reconciliation when boundaries of a hierarchically higher cluster include the boundaries of the subclusters that in turn form the boundary of a higher-level cluster. Such operation is iterative and tends to converge to the level of satisfactory description of DPCS on both levels.

For example, in case of a simplified view of a group distance learner the sub DPCS can include individual learners, local instructional materials, instructors, web sites containing learning objects, and supporting intelligent learning agents. Actors and technology in mediating roles form channels, while those in knowledge production and consumption form entities. The whole set of these DPCS forms a meta DPCS as a specific group learner. By shifting to the global vision of such group, the learner can allow to detect extra entities and channels related to group tasks and group-owned documents. This corrects the simple sum of local DPCS views. At the same time some particular local learners can play a shifting entity/channel role of learners/mediators, which adds precision to the vision of the boundaries of context and pattern clusterization in the global learner.

DPCS transformations can be performed on the designed/detected DPCS architecture. They include vertical and horizontal composition and decomposition of DPCS into sub DPCS and back to meta DPCS as well as horizontal (dis)aggregation of networked DPCS. Encapsulation and exposing of such composition/decomposition can allow for hiding or revealing the internal architecture of meta DPCS automatically allowing for collaborating actors to do the focusing on important aspects and abstracting from unnecessary details. 
Possibility to see groups of DPCS as one context cluster and deconstruct such clusters into parts is an important support for requirement 1 . Requirement 2 is additionally supported by the fact that DPCS as context clusters inherently have fuzzy permeable boundaries [4] and can shrink, expand, merge, and split. This also allows for a very flexible collaborative analysis by rolling up the vision of the architectural parts or drilling them down in an OLAP fashion. The choice of the active view should be synchronized before the beginning of learning collaborative sessions.

\section{PART 4. MEANING NEGOTIATION IN COLA}

It is not enough to be able to somehow break down the model into a number of parts matching the number of real world stabilities. Metalanguages can do it with meaning free placeholders of different size and relationships (like folders, subfolders, files, etc.). We need to be able to effectively express and control semantics/meaning of the described constructs. This was an initial problem of XML, currently partly improved by the use of semantic networks and "semanticised" schemas linked to XML files. In collaborative environments COLA uses Montagovian semantic role-function assignment in its architectural components adding the extra aspect of dynamic group/machine decision making and opinion convergence on these roles. The finite number of semantic roles together with their recursive reuse within nesting COLA models is the key to simplicity and regularity of semantic descriptions. Every component of COLA can be represented as COLA model itself and related clusters of entity/channel components can be aggregated as one integrated COLA design. Once recursive and nested COLA models are determined - they all have the same properties and self-organization dynamics thus supporting the requirement 3 .

The semantic role assignment has another specific feature in COLA. As a result of fuzzy entity/channel DPCS recognition the role differences between structural entity roles and behavioral relationship roles become also fuzzy resulting in a dynamic blend of patternstabilization roles and context-sensitivity roles for each DPCS component. For example, the perception channel can initially be seen more as a process (with fluid context) than an entity (with a stable pattern), but with continuing learning - convergence of focusing and ease of percept recognition result into a stable entity-like representation in a form of a "percept" with conceptual boundaries, pattern form, etc.

The meaning of the whole, consisting of partial meanings, can also be more of an entity type, as a structural combination of partial components, or more of a channel type, as a workflow of whole-task consisting of partial tasks. In dealing with dynamic learning situations it is important to describe the meaning of sequences of DPCS evolving in learning workflows along with such dynamic management characteristics as concurrency (sequential and parallel operations), synchronization of such processes working in parallel in different areas, and staging as possibility to describe sequencing among groups of processes (e.g. after perception is being satisfactory, negotiation of the correspondence of perceived scenes to the pre-existing knowledge can begin).

Encapsulation of implementation details of any DPCS can be done on the level of carrier abstraction [4] satisfying the fourth requirement from part 1 of this paper. Abstracting clusters of 
mutual sensitivity of their context areas from implementation details serves as an additional generative resource for DPCS based languages and models.

\section{SUMMARY}

The proposed extension of basic architectural concepts used in learning architectures allows for the development of more adaptive and general models that can be used in non-standard learning environments, situations, and with non-standard learners. We showed how these extensions allow to comply with the formulated in this paper requirements for architectural flexibility, graduality and adaptability. The concepts of active evolving clusters of context are implementation-agnostic and support the requirement of implementation independence. The finite number of COLA components combined with their descriptive richness and simple meaning negotiation can support the development of complete architectural languages. The future work can include the development of more comprehensive design and software support for COLA adaptation processes.

\section{REFERENCES}

1. Learning Technology Standards Committee. http://ltsc.ieee.org/

2. IMS Global Learning Consortium, Inc. Learning Design Specifications. Retrieved from the Web February 28, 2005. http://www.imsglobal.org/learningdesign/index.html

3. Vengerov, A. (2002a). Toward Integrated Pattern-Oriented and Case Base Design Framework in Complex Multiagent System Development for E-Business Environments. Proceedings of the Fifth Annual International Conference of ASBBS, London.

4. Vengerov A. (2003). Context-Oriented Learning Architecture. WACRA 2003 the 21st International Conference on Case Method Research and Application, Bordeaux Business School, Bordeaux, France.

5. Vengerov D., Berenji, H. \& Vengerov, A. (2002b). Adaptive coordination among fuzzy reinforcement learning agents performing distributed dynamic load balancing. 2002 IEEE International Conference on Fuzzy Systems, Honolulu, HI.

6. Vengerov A. (2005). Context - Oriented Learning Architecture (COLA). International Conference on Technology, Knowledge and Society, Berkeley, USA.

7. Brown, J.S., Collins, A. \& Duguid, P. (1989). Situated cognition and the culture of learning. Educational Researcher, (18), 32-42. Cambridge, MA: MIT Press.

8. Lave, J. \& Wenger, E. (1991). Situated learning: Legitimate peripheral participation. New York: Cambridge University Press.

9. Wilson, B., Teslow, J. \& Osman-Jouchoux, R. (1995). The impact of constructivism (and postmodernism) on ID fundamentals (pp. 137-157). In B. Seels (Ed.), Instructional design fundamentals: A review and reconsideration. Englewood Cliffs NJ: Educational Technology Publications

10. Zadeh, L. (1994). Fuzzy logic, neural networks, and soft computing. Communications of the ACM, (37), 77-84. 